\title{
Charting new frontiers in In Vitro Fertilization (IVF): The Role of Bioengineering
}

\author{
Márcia M. Carneiro ${ }^{1}$, Rívia M. Lamaita ${ }^{1,2}$, Marcos P. Barbosa $^{3}$, Agnaldo L. Silva-Filho ${ }^{1}$ \\ ${ }^{1}$ Departament of Gynecology and Obstetrics, Faculty of Medicine, Federal University of Minas Gerais, Brazil \\ ${ }^{2}$ Human Reproduction Center, Mater Dei Hospital, Belo Horizonte/MG, Brazil \\ ${ }^{3}$ Department of Mechanical Engineering, Federal University of Minas Gerais, Brazil
}

\begin{abstract}
Since the beginning of in vitro fertilization (IVF) 36 years ago, scientists have studied and critically analyzed the techniques in order to find ways to improve outcomes. However, success rates vary significantly among clinics due to poor reproducibility and inconsistency across operators. Much research has been conducted on the chemical environment, or culture medium, surrounding the oocyte/ embryo, but little attention has been given to the actual equipment and physical culture environment, which has changed very little over the years. The aim of this paper was to evaluate how the physical factors are important regulators of oocyte and embryo function and to improve understanding of the physical forces involved in the processes in human reproduction. A review the available literature was conducted using PubMed from 1966 through July 2014 in an attempt to help integrate mechanics into our understanding of the molecular basis of IVF. Keywords included in vitro fertilization, biomechanics, bioengineering, oocyte and embryo. The mechanical characterization of oocytes and embryos represents an opportunity to detect cellular defects, assess quality and bio-viability of processes such as cryopreservation as well as select the best embryo for transfer. Defining the mechanical forces at play during embryo transfer is also an important step towards improving results in in vitro fertilization. The further analysis of these phenomena needs a detailed monitoring of the mechanical conditions and more extensive studies of events on the cellular and molecular levels.
\end{abstract}

Keywords: Biomechanics, mechanical model, mechanical properties, microinjection, in vitro fertilization, oocyte.

\section{INTRODUCTION}

It has been 36 years since Patrick Steptoe and Robert Edwards announced the birth of the first in vitro fertilization (IVF) baby, Louise Brown (Steptoe \& Edwards, 1978). The development of IVF made the oocyte available to evaluation. Clearly, early biological events in the unfertilized and fertilized oocyte can have a profound effect on the later developmental stages. The development of a non-invasive score system, with emphasis on the oocyte, may help the selection of the most viable single embryo for transfer (Scott, 2003; Combelles \& Racowsky, 2005).

In spite of the great technological advancement in IVF, little has changed with the present technology and equipment available despite the large number of studies performed worldwide in an attempt to find ways to achieve better outcomes in terms of pregnancy and live-birth rates. Much of the attention has focused on the oocyte/embryo and the surrounding culture medium but little consideration has been given to the actual equipment and biophysical culture environment, which has improved but changed very little over the years (Yaniv et al., 2009; Smith \& Monteiro da Rocha, 2012; Smith et al., 2012; Swain et al., 2013).

A number of biophysical factors are important regula- tors of gamete and embryo function, but improved understanding of the physical forces involved in the processes in human reproduction requires novel experimental platforms. In order to bridge this gap, engineers are building tools to control mechanical factors with improved precision and throughput, thereby enabling biological investigation of mechanics-driven function in an attempt to improve understanding and IVF results (Smith et al., 2012; Swain et al., 2013; Heo et al., 2012).

Mechanobiology is an emerging field which focus on the application of biophysics or biomechanics in an attempt to unravel and explain biological and physiological functions in living organism at the various levels ranging from cells to organs. This encompasses molecular interactions at the DNA which control gene transcription as well as the forces that control cell migration, adhesion and transport of various materials between and within cells. Consequently, the interplay between these molecular and cellular forces interferes in the conformation of cells and tissues and consequently in their physiology. As mechanobiology allows the understanding of molecular, cellular and tissue functioning it will not only establish but open new frontiers of knowledge and understanding in the biomedical sciences research (Ingber, 2003; Eyckmans et al., 2011).

Mechanotransduction depicts the cellular processes that decode mechanical stimuli into biochemical signals, thus allowing cells to accommodate to their physical surroundings. The translation of these mechanical forces and deformations into biochemical signals is called mechanotransduction, which could result, for example, in alterations in intracellular calcium concentration or incitement of numerous signaling pathways. The activation of such signals can result in adjustment of cellular and extracellular structure. Diverse cellular functions such as migration, proliferation, differentiation and apoptosis are regulated by his mechanosensitive feedback which is fundamental for organ development and homeostasis (Mammoto et al., 2012).

Microfluidics is a novel technology dealing with the behavior of liquids in a microenvironment. In spite of being a recent developed field, a great deal of research corroborates the potential of this technology in gamete and embryo isolation and culture which casts a new look on the way IVF is performed. Much progress has been made in the area of microfluidic systems for in vitro embryo production and embryo manipulation which could have a significant impact on the development of new techniques as well as on our fundamental knowledge of gamete and embryo physiology (Smith et al., 2007; Wheeler et al., 2007). IVF is a labor-intensive process involving controlled ovarian hyperstimulation ( $\mathrm{COH})$, oocyte pickup (OPU), oocyte fertilization and embryo transfer (ET) as well as embryo cryopreservation. Usually these procedures are tightly controlled via hormonal and ultrasonographic parameters. However, the physical environment surrounding gametes and embryos or the uterus itself has not been adequately considered so far. Recently, research has begun to focus on 
the physical requirements of all the steps involved in IVF which could influence the results (Yaniv et al., 2009; Smith \& Monteiro da Rocha, 2012; Wheeler et al., 2007; Morton et al., 2008).

Thousands of couples depend upon IVF to achieve a pregnancy. In spite of all efforts and technological advancements, results in terms of live-birth rates remain low. Thereby, the main goal of this article is to review the available literature on the subject in an attempt to help integrate mechanics into our understanding of the molecular basis of IVF as physical forces, extracellular matrix and cell structure play a key role in the control of normal development, as well as in the maintenance of tissue homeostasis. Thus understanding the mechanical forces at play in IVF may aid us in obtaining better results. A review the available literature was conducted using PubMed from 1966 through July 2014 in an attempt to help integrate mechanics into our understanding of the molecular basis of IVF. Keywords included in vitro fertilization, biomechanics, bioengineering, oocyte and embryo.

\section{Aspiration of oocytes}

Oocyte aspiration is usually performed with the aid of transvaginal ultrasonography under local anesthesia or sedation in order to obtain oocytes for in vitro fertilization. Such technique permits considerable simplification of egg retrieval for IVF, which can now be performed in an outpatient setting eliminating the risks and costs of laparoscopy and general anesthesia (Dellenbach et al., 1985). Initially, a manual syringe suction system was used to aspirate follicles. The use of such as system was associated a significant increase in the proportion of oocytes with fractured zona pellucida (ZP), a lower normal fertilization rate and a higher proportion of multipronuclear fertilizations. It was concluded that the aspiration pressures, created by syringe suction, were more likely to rupture the ZP of some oocytes, while in others it predisposed to an increased multipronuclear fertilization rate. Thus the manual system was abandoned by many in favor of an electric aspiration pump though some still use it (Cohen et al., 1986; Lowe et al., 1988).

Except for a comparison of manual and mechanical suction on the effect of zonal damage there has been strangely little published on the theory of oocyte aspiration and retrieval much less on the mechanical and biophysical forces involved in the procedure (Horne et al., 1996). Animal studies confirm the influence of various biophysical variables such as aspiration pressure during oocyte retrieval on the number of oocytes obtained, fertilization rates and embryo development (Morton et al., 2008; Brüssow et al.; 1997; Fry et al., 1997; Rodríguez et al., 2006). Human studies, on the other hand are scarce.

In one of the very few studies available in humans, Hashimoto et al. (2007) evaluated the effects of various aspiration vacuums during oocyte retrieval with a 20-gauge single lumen needle on the developmental capability of immature oocytes after in vitro maturation (IVM), IVF and embryo transfer (Hashimoto et al., 2007). Immature oocytes were aspirated by 20 -gauge single lumen needle with a pressure of 180 or $300 \mathrm{mmHg}$. The number of retrieved oocytes and the developmental rate of oocytes to transferable embryos increased using the lower aspiration vacuum.

\section{Oocyte assessment}

Despite all the recent advancements in IVF technology, morphological oocyte evaluation, although extremely subjective, remains the basic criterion generally used in intracytoplasmic sperm injection (ICSI) (Wang et al., 2007; Valeri et al., 2011). Oocyte morphological evaluation before ICSI helps to identify metaphase II (MII) oocytes appropriate for sperm injection which show increased developmental capacity. Such evaluation includes comprises variations of oocyte form, color, granularity and homogeneity of cytoplasm, width of perivitelline space, debris in the perivitelline space, vacuolization, inclusions and abnormalities of the first polar body $\left(1^{\text {st }} \mathrm{PB}\right.$ ) or of the ZP (Wilding et al., 2007; La Sala et al., 2009; Levi et al., 2013).

However, still today determining oocyte quality remains a challenge. Developing a better understanding of physiological events involved in oocyte development is needed to protect fertility, fecundity, and ovarian function in women as oocyte quality is a key factor affecting success of human IVF (Valeri et al., 2011; Braga et al., 2013; Kurus et al., 2013). Since maternal age is of paramount importance in predicting pregnancy and live birth, the identification of aged-related indicators of oocyte quality is of considerable interest in the field of IVF (Stensen et al., 2010). Thus the search for reliable predictors of oocyte developmental competence drives the continued research for more precise and objective markers. In most cases, however, embryologists select them by visual examination and their evaluation is totally subjective (Wang et al., 2007; Wilding et al., 2007).

The mechanical characterization of a living cell involves cell deformation in some way and measurement of the applied forces/stresses. The advancement of micromanipulation technology has allowed the development of some experimental techniques capable of measuring the mechanical properties of cells, such as micropipette aspiration (Lie et al., 2010).

There are very few published papers concerning the mechanical behavior of human oocytes. According to animal studies, the stiffness of the oocyte varies during the meiotic maturation. Such variations in oocyte stiffness can be attributed two mechanisms: the change of the ZP thickness during the maturation of the oocyte and the formation of actin filaments resulting in a thin network in different region of the oocyte (Sun et al., 2003).

The ZP is an extracellular membrane enclosing mammalian oocytes. The so-called zona hardening plays a key role in fertilization process, as it blocks polyspermy, which may also be caused by an increase in the mechanical stiffness of the ZP membrane (Sun et al., 2003). However, structural reorganization mechanisms leading to ZP's biomechanical hardening are not fully understood.

Murayama et al. (2006) quantitatively evaluated the change in the elasticity of mouse ZP during oocyte maturation, fertilization and early embryo development [33]. The mouse ZP hardened following fertilization, but, it gradually softened as the embryo developed. Furthermore, the ZP of the germinal vesicle (GV) oocyte was as hard as that of the PN embryo and became soft as it matured to the MII stage. These authors recently published paper on the change in the elasticity and the resistance to dissolution of the mouse ZP at GV, mature metaphase II (MII) and fertilized pronuclear (PN) stages. After measuring the ZP elasticity and the dissolution time, they clearly showed that the ZP softened during oocyte maturation and hardened after fertilization. The results indicate that the amount of the zona softening can be a criterion to evaluate oocyte quality for the selection of top quality mature oocyte before IVF.

Boccaccio et al. (2012) investigated the biomechanical behavior of ZP membranes extracted from mature and fertilized bovine oocytes to better understand the mechanisms involved in the structural reorganization of the ZP that may lead to the biomechanical hardening of the ZP. Apparently, fertilization leads to an increase in the degree of heterogeneity of membrane elastic properties. These findings support the hypothesis that biomechanical hardening of bovine ZP is caused by an increase in the number of inter-filaments cross-links whose density should be 
higher in the ZP inner side.

Wacogne et al. (2008) built a system to evaluate human oocyte mechanical properties (elasticity). The system consisted of an SU-8 micro-beam which acted as a force sensor. When pressed onto the micro-beam, the oocyte undergoes deformation. At the same time the micro-beam bends. The knowledge of both, the stiffness and the deflection of the beam, allows drawing the "deformation load" curve corresponds to the cell under test. Oocyte deformation and the beam deflection are measured by means of image processing and data are computed to estimate some mechanical characteristics of the oocytes. This image processing can directly be used by biologists or clinical embryologists with their standard IVF micro-manipulators.

Liu et al. (2010) developed a cellular force measurement technique that allows for mechanical characterization of mouse oocytes during microinjection. Their experimental results suggest that the in situ obtained force-deformation data are useful for distinguishing healthy mouse oocytes from those with aging-induced cellular defects, promising an approach for oocyte quality assessment during microinjection. These authors went further and demonstrated that aged oocytes are significantly softer but more viscous than the young oocytes (Liu et al., 2012). No published studies on human oocytes exist so far

It is clear from the data above that the subject of oocyte assessment still needs further studies as most published papers concern animal oocytes and use different methodologies which does not allow for a definite conclusion.

\section{Embryo quality}

Extend in-vitro culture and blastocyst transfer are useful proceduresused in assisted reproduction. This procedure permitted the selection of embryos at more advanced stages of development, where the surviving embryos are more likely to produce a successful result. The estimation of embryo viability prior to transfer is notoriously subjective, with morphological traits such as nucleation, cell numbers, fragmentation and cell size distribution being the most commonly used parameters for selection (Scholtes et al., 1996). The reliable selection of viable embryos for transfer is a key factor for determining the success of IVF. However, the attributes of 'optimal embryos' and the methods to identify embryos with a high developmental potential are still unknown entities (Cruz et al., 2012). In most cases, embryologists select embryos by visual examination and their evaluation is totally subjective and has several limitations (Manna et al., 2013; Montag et al., 2013; Santos Filho et al.; 2012, Freour et al., 2012).

The recent development of clinically applicable real-time imaging systems to assess embryo morphokinetics, has sparked renewed interest regarding noninvasive methods to assess gamete and embryo development as a means of inferring quality. Several studies exist that utilize novel imaging techniques to visualize or quantify intracellular components of gametes and embryos with the intent of correlating localization of organelles or molecular constitution with quality or outcome (Manna et al., 2013; Jasensky et al., 2011).

A study introduced specific temporal development markers that were related with subsequent implantation. The authors evaluated the chronological pattern of cell divisions as well as other morphological features (cell size and nucleation) in order to reveal connections between the time taken to reach each developmental milestone and the implantation potential of the specific embryo. They identified an optimal range for each morphokinetic variable which was associated with a significantly higher probability of implantation. The data indicate that embryos that cleave earlier have a significantly improved chance of continuing development to day 5 when compared with embryos that develop more slowly (Meseguer et al., 2011).

Recently, some studies examined the cytoplasmic movements triggered by sperm entry, Ca2+ oscillations, dynamics of zygote shape and cytoskeleton function in order to examine whether the dynamics of these movements can be used to predict developmental success. These experiments related to cytoplasm movements not only help to predict blastocyst development but also embryo quality, since they could establish a significant linear relationship between mean basal speed of cytoplasm movements and the cell count in an embryo (Ajduk et al., 2011; Swann et al., 2012).

A clear advantage of cleavage-stage embryo transfer, besides the associated labor savings, is the shortened exposure of the embryo to artificial in-vitro conditions where imprinting changes could cause adverse epigenetic problems. Quite possibly to the extent that blastocyst culture may no longer be needed. Therefore, it may well be that the morphokinetic parameters that predict blastocyst formation could differ from those that predict implantation (Wong et al., 2010).

\section{Embryo transfer}

Embryo transfer (ET) is the final manual intervention in IVF in which an embryo is transferred into the uterus by a transcervical catheter. The low rates of embryo implantation within the uterus are attributed, among other factors, to the ET technique, which depends on a multitude of anatomical, physiological, and mechanical aspects (Eytan et al., 2004).

Uterine contractility may influence the human embryo implantation process in both natural and stimulated cycles. Such contractions are under the influence of the ovarian steroids and, supposedly, partake in the IVF and embryo implantation processes in humans. Approaches aimed at understanding and controlling uterine contractions could be pivotal in improving pregnancy rates after the use of assisted reproductive technologies (Bulletti \& de Ziegler, 2006, Fanchin \& Ayoubi, 2009 )

The injection velocity greatly influences the trajectories of the embryos, and it may determine their acceleration, to which site they will be transported and how influential will be the peristaltic motion on ET. Understanding of intrauterine transport characteristics during and after the ET maneuver, as well as the delicate interaction between uterine peristalsis and the controlling parameters of ET, may lead to improved procedures with a better outcome of IVF (Yaniv et al., 2003).

Eytan et al. (2007) developed computational and experimental models to simulate ET to study the contribution of mechanical features to the success of this procedure. The experimental model allowed laboratory simulations of the dispersion of the catheter load as a result of different injection speeds into a tilted uterine model. They concluded that the mechanical parameters such as the position of the uterus and the presence of air in the catheter load are important parameters for the success of ET.

According to Yaniv et al. (2009) embryos are expected to be implanted in the uterine wall in the vicinity where they were initially placed, either naturally or artificially, within the uterine cavity.

Yaniv et al. (2012) tried to establish the role of intrauterine fluid flow patterns in transporting the embryos to their implantation site during and after ET by simulating the injection of a liquid bolus into a two-dimensional liquid-filled channel with a closed fundal end via a liquid-filled catheter inserted in the channel. Numerical experiments revealed that the intrauterine fluid field and the embryos transport pattern were strongly affected by the closed fundal end. The embryos re-circulated in small loops around the vicinity where they were deposited from the cathe- 
ter. The transport pattern was controlled by the uterine peristalsis factors, such as amplitude and frequency of the uterine walls motility, as well as the synchronization between the onset of catheter discharge and uterine peristalsis. Clearly these mechanical parameters should be taken into consideration so as to improve IVF results.

\section{CONCLUSIONS}

Oocyte and embryo quality assessment are an important procedure to identify the healthiest oocytes for IVF as well as the embryos with the highest reproductive potential for implantation. However even now, gamete/embryo morphology and cleavage rates are the most common form of quality assessment. Thus, still today, embryologists rely on a totally subjective evaluation. In IVF, the mechanical characterization of oocytes and embryos represents an opportunity to detect cellular defects, assess quality and bio-viability of processes such as cryopreservation as well as select the best embryo for transfer. Defining the mechanical forces at play during embryo transfer is also an important step towards improving results in IVF. In spite of all the evidence pointing to its importance, this area is still largely underexplored.

\section{CONFLICT OF INTERESTS}

No conflict of interest have been declared.

\section{Corresponding author:}

Agnaldo Lopes da Silva Filho

Department of Obstetrics and Gynecology

Federal University of Minas Gerais (UFMG)

Avenida Professor Alfredo Balena, 190

Santa Efigênia - Belo Horizonte/MG - Brazil

E-mail: agnaldo.ufmg@gmail.com

\section{REFERENCES}

Ajduk A, Ilozue T, Windsor S, Yu Y, Seres KB, Bomphrey RJ, Tom BD, Swann K, Thomas A, Graham C, Zernicka-Goetz M. Rhythmic actomyosin-driven contractions induced by sperm entry predict mammalian embryo viability. Nat Commun. $2011 ; 2: 417$

Boccaccio A, Frassanito MC, Lamberti L, Brunelli R, Maulucci G, Monaci M, Papi M, Pappalettere C, Parasassi T, Sylla L, Ursini F, De Spirito M. Nanoscale characterization of the biomechanical hardening of bovine zona pellucida. J R Soc Interface. 2012;9:2871-82

Braga DP, Setti AS, Figueira R de C, Machado RB, Iaconelli A Jr, E Borges Jr. Influence of oocyte dysmorphisms on blastocyst formation and quality. Fertil Steril. 2013,100:74854.

Brüssow KP, Torner H, Rátky J, Hunter MG, Nürnberg G. Ovum pick up in swine: the influence of aspiration vacuum pressure on oocyte recovery from preovulatory follicles. Acta Vet Hung. 1997;45:189-96.

Bulletti C, de Ziegler D. Uterine contractility and embryo implantation. Curr Opin Obstet Gynecol. 2006;18:473-84.

Cohen J, Avery S, Campbell S, Mason BA, Riddle A, Sharma V. Follicular aspiration using a syringe suction system may damage the zona pellucida. J In Vitro Fert Embryo Transf. 1986;3:224-6.

Combelles CM, Racowsky C. Assessment and optimization of oocyte quality during assisted reproductive technology treatment. Semin Reprod Med. 2005; 23:277-84.

Cruz M, Garrido N, Herrero J, Pérez-Cano I, Muñoz
M, Meseguer M. Timing of cell division in human cleavage-stage embryos is linked with blastocyst formation and quality. Reprod Biomed Online. 2012;25:371-81.

Dellenbach P, Nisand I, Moreau L, Feger B, Plumere C, Gerlinger P. Transvaginal sonographically controlled follicle puncture for oocyte retrieval. Fertil Steril. 1985;44:65662.

Eyckmans J, Boudou T, Yu X, Chen CS. A hitchhiker's guide to mechanobiology. Dev Cell. 2011; 21:35-47.

Eytan O, Elad D, Zaretsky U, Jaffa AJ. A glance into the uterus during in vitro simulation of embryo transfer. Hum Reprod. 2004;19:562-9.

Eytan O, Elad D, Jaffa AJ. Bioengineering studies of the embryo transfer procedure. Ann N Y Acad Sci. 2007;1101:2137.

Fanchin R, Ayoubi JM. Uterine dynamics: impact on the human reproduction process. Reprod Biomed Online. 2009;18 Suppl 2:57-62.

Freour T, Lammers J, Splingart C, Jean M, Barriere P. [Time lapse (Embryoscope $\AA$ ) as a routine technique in the IVF laboratory: a useful tool for better embryo selection?]. Gynecol Obstet Fertil. 2012;40:476-80.

Fry RC, Niall EM, Simpson TL, Squires TJ, Reynolds J. The collection of oocytes from bovine ovaries. Theriogenology. 1997;47:977-87.

Hashimoto S, Fukuda A, Murata $Y$, Kikkawa M, Oku H, Kanaya $\mathrm{H}$, Sonoda $\mathrm{M}$, Sugihara $\mathrm{K}$, Murata $\mathrm{T}$, Nagata $\mathrm{F}$, Nakaoaka $Y$, Morimoto $Y$. Effect of aspiration vacuum on the developmental competence of immature human oocytes retrieved using a 20-gauge needle. Reprod Biomed Online. 2007; 14:444-9.

Heo YS, Cabrera LM, Bormann CL, Smith GD, Takayama S. Real time culture and analysis of embryo metabolism using a microfluidic device with deformation based actuation. Lab Chip. 2012;12:2240-6.

Horne R, Bishop CJ, Reeves G, Wood C, Kovacs GT. Aspiration of oocytes for in-vitro fertilization. Hum Reprod Update. 1996;2:77-85.

Ingber DE. Mechanobiology and diseases of mechanotransduction. Ann Med. 2003, 35:564-77.

Jasensky J, Swain JE. Peering beneath the surface: novel imaging techniques to noninvasively select gametes and embryos for ART. Biol Reprod. 2013; 89:105.

Kurus M, Karakaya C, Karalok MH, To G, Johnson J. The control of oocyte survival by intrinsic and extrinsic factors. Adv Exp Med Biol. 2013; 761:7-18.

La Sala GB, Nicoli A, Villani MT, Di Girolamo R, Capodanno $\mathrm{F}$, Blickstein I. The effect of selecting oocytes for insemination and transferring all resultant embryos without selection on outcomes of assisted reproduction. Fertil Steril. 2009; 91:96-100.

Levi M, Ghetler Y, Shulman A, Shalgi R. Morphological and molecular markers are correlated with maturation-competence of human oocytes. Hum Reprod. 2013; 28: 2482-9.

Liu X, Fernandes R, Jurisicova A, Casper RF, Sun Y. In situ 
mechanical characterization of mouse oocytes using a cell holding device. Lab Chip. 2010; 10:2154-61.

Liu X, Shi J, Zong Z, Wan KT, Sun Y. Elastic and viscoelastic characterization of mouse oocytes using micropipette indentation. Ann Biomed Eng. 2012; 40:2122-30.

Lowe B, Osborn JC, Fothergill DJ, Lieberman BA. Factors associated with accidental fractures of the zona pellucida and multipronuclear human oocytes following in-vitro fertilization. Hum Reprod. 1988; 3:901-4.

Mammoto A, Mammoto T, Ingber DE. Mechanosensitive mechanisms in transcriptional regulation. J Cell Sci. 2012;125:3061-73.

Manna C, Nanni L, Lumini A, Pappalardo S. Artificial intelligence techniques for embryo and oocyte classification. Reprod Biomed Online. 2013; 26:42-9.

Meseguer M, Herrero J, Tejera A, Hilligsøe KM, Ramsing NB, Remohí J. The use of morphokinetics as a predictor of embryo implantation. Hum Reprod. 2011; 26:2658-71.

Montag M, Toth B, Strowitzki T. New approaches to embryo selection. Reprod Biomed Online. 2013;27:539-46.

Morton KM, Maxwell WM, Evans G. Effect of aspiration pressure during oocyte harvesting on oocyte recovery and in vitro development of ovine oocytes. Reprod Domest Anim. 2008; 43:106-10.

Murayama $\mathrm{Y}$, Mizuno J, Kamakura $\mathrm{H}$, Fueta $\mathrm{Y}$, Nakamura $\mathrm{H}$, Akaishi K, Anzai K, Watanabe A, Inui H, Omata S. Mouse zona pellucida dynamically changes its elasticity during oocyte maturation, fertilization and early embryo development. Hum Cell. 2006; 19:119-25.

Rodríguez C, Anel L, Alvarez M, Anel E, Boixo JC, Chamorro CA, de Paz P. Ovum pick-up in sheep: a comparison between different aspiration devices for optimal oocyte retrieval. Reprod Domest Anim. 2006; 41:106-13.

Santos Filho E, Noble JA, Poli M, Griffiths T, Emerson G, Wells D. A method for semi-automatic grading of human blastocyst microscope images. Hum Reprod. 2012;27:2641-8.

Scholtes MC, Zeilmaker GH. A prospective, randomized study of embryo transfer results after 3 or 5 days of embryo culture in in vitro fertilization. Fertil Steril. 1996; $65: 1245-8$.

Scott L. The biological basis of non-invasive strategies for selection of human oocytes and embryos. Hum Reprod Update. 2003; 9:237-49.

Smith GD, Monteiro da Rocha A. Advances in embryo culture systems. Semin Reprod Med. 2012; 30:214-21.

Smith GD, Takayama S. Gamete and embryo isolation and culture with microfluidics. Theriogenology. 2007; 68 Suppl 1: S190-5.

Smith GD, Takayama S, Swain JE. Rethinking in vitro embryo culture: new developments in culture platforms and potential to improve assisted reproductive technologies. Biol Reprod. 2012; 86:62.

Stensen $\mathrm{MH}$, Tanbo $\mathrm{T}$, Storeng R, Byholm T, Fèdorcsak P. Routine morphological scoring systems in assisted reproduction treatment fail to reflect age-related impairment of oocyte and embryo quality. Reprod Biomed Online. 2010; 21:118-25.

Steptoe PC, Edwards RG. Birth after the reimplantation of a human embryo. Lancet. 1978; 2:366.

Sun Y, Wan KT, Roberts KP, Bischof JC, Nelson BJ. Mechanical property characterization of mouse zona pellucida. IEEE Trans Nanobioscience. 2003; 2:279-86.

Swain JE, Lai D, Takayama S, Smith GD. Thinking big by thinking small: application of microfluidic technology to improve ART. Lab Chip. 2013; 13:1213-24.

Swann K, Windsor S, Campbell K, Elgmati K, Nomikos M, Zernicka-Goetz M, Amso N, Lai FA, Thomas A, Graham C. Phospholipase C-zeta-induced $\mathrm{Ca} 2+$ oscillations cause coincident cytoplasmic movements in human oocytes that failed to fertilize after intracytoplasmic sperm injection. Fertil Steril. 2012; 97:742-7.

Valeri C, Pappalardo S, De Felici M, Manna C. Correlation of oocyte morphometry parameters with woman's age. J Assist Reprod Genet. 2011; 28:545-52.

Wacogne B, Pieralli C, Roux C, Gharbi T. Measuring the mechanical behaviour of human oocytes with a very simple SU-8 micro-tool. Biomed Microdevices. 2008; 10:411-9.

Wang Q, Sun QY. Evaluation of oocyte quality: morphological, cellular and molecular predictors. Reprod Fertil Dev. 2007; 19: 1-12.

Wheeler MB, Walters EM, Beebe DJ. Toward culture of single gametes: the development of microfluidic platforms for assisted reproduction. Theriogenology. 2007; 68 Suppl 1: S178-89.

Wilding M, Di Matteo L, D'Andretti S, Montanaro N, Capobianco C, Dale B. An oocyte score for use in assisted reproduction. J Assist Reprod Genet. 2007; 24:350-8.

Wong CC, Loewke KE, Bossert NL, Behr B, De Jonge CJ, Baer TM, Reijo Pera RA. Non-invasive imaging of human embryos before embryonic genome activation predicts development to the blastocyst stage. Nat Biotechnol. $2010 ; 28: 1115-21$.

Yaniv S, Elad D, Jaffa AJ, Eytan O. Biofluid aspects of embryo transfer. Ann Biomed Eng, 2003;31: 1255-62.

Yaniv S, Jaffa AJ, Eytan O, Elad D. Simulation of embryo transport in a closed uterine cavity model. Eur J Obstet Gynecol Reprod Biol. 2009,144 Suppl 1: S50-60.

Yaniv S, Jaffa AJ, Elad D. Modeling embryo transfer into a closed uterine cavity. J Biomech Eng. 2012; 134: 111003. 